Arq. Bras. Med. Vet. Zootec., v.66, n.2, p.381-387, 2014

\title{
Sistemas de efluxo multidrogas em Escherichia coli e uso de inibidores como possíveis adjuvantes na terapia da mastite bovina
}

\author{
[Multidrug efflux systems in Escherichia coli and the use of inhibitors as possible \\ adjuvant therapy in bovine mastitis]
}

\author{
M.A. Ospina, F.A. Pieri, P.A. Pietralonga, M.A.S. Moreira* \\ Universidade Federal de Viçosa - UFV - Viçosa, MG
}

\begin{abstract}
RESUMO
Vinte e sete isolados de Escherichia coli provenientes de leite de bovinos com mastite clínica foram submetidos a teste de sensibilidade aos principais antimicrobianos usados no tratamento desta doença. Avaliou-se também a efetividade in vitro de dois inibidores de sistemas de efluxo multidrogas, fenilalanina arginyl $\beta$ naftilamida $(\mathrm{PA} \beta \mathrm{N})$ e 1-(1-Naphthylmethyl)-piperazine (NMP), utilizando-se a concentração inibitória mínima (CIM) como referência. A CIM e o sistema de efluxo foram detectados com base nas curvas de crescimento, utilizando-se a densidade óptica (D. $\mathrm{O}^{550}$ ), em diferentes concentrações da droga e na presença e ausência dos inibidores. Apenas quatro isolados apresentaram resistência à ampicilina e ao sulfametoxazo/trimetoprim, simultaneamente, enquanto todos os 27 foram sensíveis aos demais antimicrobianos. Observaram-se valores para ampicilina variando de $6 \mu \mathrm{g} / \mathrm{mL}$ a $250 \mu \mathrm{g} / \mathrm{mL}$ e para sulfametoxazoltrimetoprim de $12 \mu \mathrm{g} / \mathrm{mL}$ a $1500 \mu \mathrm{g} / \mathrm{mL}$. Houve redução das CIMs desses antimicrobianos em todos os isolados na presença dos inibidores, exceto para sulfametoxazo/trimetoprim na presença de NMP. Conclui-se que esses isolados possuem um estreito perfil de resistência e que $\mathrm{PAßN}$ apresentou melhor efeito inibitório em relação à ampicilina e ao sulfametoxazoltrimetoprim, apresentando-se como um candidato a adjuvante no tratamento da mastite.
\end{abstract}

Palavras-chave: multirresistência, bactéria, leite, mecanismo de resistência

\begin{abstract}
Twenty-seven isolates of Escherichia coli from cattle with clinical mastitis were subjected to sensitivity tests regarding main antimicrobials used in the treatment of this disease. We also evaluated in vitro effectiveness of two inhibitors of multidrug efflux systems, fenilalanina arginyl $\beta$ naftilamida (PA $\beta N)$ and 1-(1-Naphthylmethyl)-piperazine (NMP), using the minimum inhibitory concentration (MIC) as a reference. MIC and multidrug efflux systems were detected in the growth curves, using optical density $\left(D . O^{550}\right)$ at different drug concentrations and the presence and absence of inhibitors. Only four isolates of E. coli were resistant to ampicillin and trimethoprim/sulfamethoxazole, simultaneously. However, all isolates were sensitive to the other antimicrobials. Were observed values ranging from 6mg to $250 \mathrm{mg}$ ampicillin $/ \mathrm{mL}$, and $12 \mathrm{mg}$ to $1500 \mathrm{mg} / \mathrm{mL}$ trimethoprim/sulfamethoxazole. There was a reduction of the MIC of antimicrobials for all isolates in the presence of inhibitors, except for trimethoprim/sulfamethoxazole in the presence of NMP. In conclusion, these isolates have a narrow resistance profile and $P A \beta N$ showed better inhibitory effect against ampicillin and trimethoprim/sulfamethoxazole, and is a candidate for the adjuvant treatment of mastitis.
\end{abstract}

Keywords: multirresistance, bacteria, milk, resistance mechanism

Recebido em 3 de julho de 2012

Aceito em 6 de setembro de 2013

*Autor para correspondência (corresponding author)

E-mail: masm@ufv.br 


\section{INTRODUÇÃO}

A mastite bovina é uma das doenças mais importantes na indústria leiteira, causa grandes perdas econômicas no setor lácteo, estimadas na ordem de 35 bilhões de dólares anuais em todo o mundo (Huijps et al., 2009). Essas perdas são ocasionadas pela redução da produção; pelo comprometimento da qualidade do leite e derivados em decorrência da alteração dos seus componentes nutricionais; pela perda parcial ou total da capacidade secretória da glândula mamária; ou mesmo pelo abate de animais (Costa, 2009). Além desses prejuízos, há ainda um risco potencial à saúde pública, visto que a disseminação de toxinas e patógenos causadores de zoonoses, via leite e derivados, é um risco, principalmente em nichos de mercado de produtos lácteos não pasteurizados, no comércio de leite informal ou durante falhas no processo de pasteurização (Altalhi et al., 2009).

Com relação à origem, a mastite pode ser classificada como contagiosa ou ambiental. O patógeno mais importante deste último tipo de mastite é Escherichia coli. Esta invade a glândula mamária através do canal do teto, por meio do contato dos tetos com o ambiente contaminado. Após a invasão, o agente pode se multiplicar ou permanecer em latência, sem causar sinais clínicos, por dias (Blum et al., 2008; Bradley e Green, 2009). Alguns isolados de E. coli possuem uma habilidade maior em aderir ao tecido mamário e nele replicar, o que pode determinar infecções persistentes no hospedeiro (Fontaine et al., 2006). Nos últimos anos, casos de mastite ambiental vêm aumentando em muitos países, geralmente em rebanhos em que houve sucesso no controle da mastite contagiosa (Oliver et al., 2011). Tal fato pode estar relacionado a falhas na terapia, resultando em infecções persistentes na glândula mamária, tanto no período de lactação quanto no período seco (Ribeiro et al., 2006; Biggs, 2009). Essas falhas podem ser atribuídas a diversos fatores, como o uso incorreto de antimicrobianos, a presença de biofilmes na superfície do tecido da glândula mamária e os mecanismos de resistência, entre os quais os sistemas de efluxo multidrogas (MDR) das bactérias. (Melchior et al., 2006; Costa, 2009).

Sistemas de efluxo multidrogas (MDR) são mecanismos desenvolvidos por algumas bactérias com a finalidade de expelir moléculas de antimicrobianos, reduzindo as concentrações da droga dentro da célula, além de outras substâncias tóxicas, como metais pesados, antissépticos e quimioterápicos (Webber e Piddock, 2003). Estudos indicam que esses sistemas ainda interferem na entrada de nutrientes na célula bacteriana, na manutenção da homeostase, na mediação da adesão bacteriana e na adaptação das bactérias às células do hospedeiro, os quais são importantes fatores de virulência (Piddock, 2006; Ricci e Piddock, 2009).

O uso de inibidores de sistemas de efluxo (EPIs) para inibir MDR em bactérias pode ser uma alternativa promissora no tratamento da mastite. Os EPIs ajudam a aumentar a concentração intracelular de antimicrobianos que são expelidos por sistemas de efluxo multidrogas (Thorarensen, 2001; Lomovskaya e Bostian, 2006) e, assim, restabelecem a sensibilidade às drogas de cepas clínicas resistentes, obtendo êxito no tratamento da doença. Há diferentes tipos de EPIs, entre eles compostos naturais e análogos de substratos de antimicrobianos e novas moléculas químicas (Viveiros et al., 2008; Matsumoto et al., 2011). De um modo geral, os inibidores atuam por competição com os antimicrobianos (Murakami et al., 2006) e alguns aumentam a permeabilidade da membrana externa (Matsumoto et al., 2011).

Em razão da grande importância da mastite nos âmbitos social, econômico e de saúde pública e do sistema de efluxo multidrogas como um mecanismo de resistência emergente em bactérias, objetivou-se avaliar a resistência e a multirresistência em E. coli, isoladas de leite provenientes de bovinos com mastite, aos antimicrobianos utilizados no tratamento dessa doença e estudar a efetividade in vitro de dois inibidores de sistemas de efluxo multidrogas.

\section{MATERIAL E MÉTODOS}

Foram utilizados 27 isolados de Escherichia coli obtidos de leite provenientes de bovinos com mastite (Fernandes et al., 2011). Os isolados foram armazenados em microtubos contendo caldo de infusão de cérebro e coração BHI (Oxoid), acrescidos de $20 \%$ de glicerol e estocados a $-80^{\circ} \mathrm{C}$. 
O antibiograma foi realizado seguindo as normas preconizadas pelo Clinical and Laboratory Standards Institute (CLSI, 2008). Os isolados de E. coli foram reativados em caldo Mueller Hinton e incubados a $37^{\circ} \mathrm{C}$, durante $16-18$ horas. As culturas foram diluídas em caldo Mueller Hinton, a $10^{8} \mathrm{UFC} / \mathrm{mL}$, turbidez equivalente a 0.5 na escala de MacFarland. Os discos de antimicrobianos foram distribuídos sobre as placas, e posteriormente estas foram incubadas a $37^{\circ} \mathrm{C}$, durante $16-18$ horas, em duplicatas. Após esse período, foi realizada a leitura dos diâmetros da média dos halos de inibição, e estes foram interpretados de acordo com os critérios preconizados pelo CLSI (2008). Os antimicrobianos utilizados foram os de uso frequente no tratamento da mastite clínica:

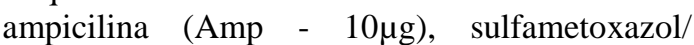

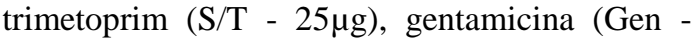
$10 \mu \mathrm{g})$, ceftiofur (Cef - 30 $\mu \mathrm{g}$ ), cefalaxina (Cen $30 \mu \mathrm{g})$, enrofloxacina (Enr - 5 $\mu \mathrm{g})$, (OXOID).

Para a determinação da concentração inibitória mínima (CIM) e do sistema de efluxo multidrogas, foi seguida a metodologia de Moreira et al. (2005) e CLSI (2008). Para isso, $230 \mu \mathrm{L}$ de cada cultura foram reativados em caldo Mueller Hinton, diluídos a $10^{5} \mathrm{UFC} \mathrm{mL}^{-1}$ e, então, adicionados à placa de microtitulação, juntamente com os antimicrobianos, nas diferentes concentrações, em um total de $300 \mu \mathrm{L}$ por orifício. O crescimento dos isolados foi acompanhado em aparelho leitor de absorbância, e as leituras das D. O $_{550}$ foram feitas em intervalos preestabelecidos até a estabilização dos valores. A curva de crescimento foi construída com a média aritmética das triplicatas. A CIM foi estabelecida como a menor concentração do antimicrobiano que impedia o crescimento bacteriano até duas horas após o tempo correspondente ao início da fase estacionária do controle (cada isolado cultivado em caldo Mueller Hinton sem antimicrobiano). Para detecção do sistema de efluxo multidrogas, foram utilizados dois EPIs, fenilalanina arginyl B naftilamida $(\mathrm{PA} \beta \mathrm{N})$ e 1-(1-Naphthylmethyl)piperazine (NMP). Previamente, foram realizados testes com diversas concentrações dos EPIs em cada isolado para conferir que esses inibidores não alterariam o crescimento dos isolados. As concentrações dos EPIs utilizadas foram com base no estudo realizado por Kern et al. (2006). Empregaram-se $50 \mu \mathrm{g} / \mathrm{mL}$ de PA $\beta \mathrm{N}$ e $75 \mu \mathrm{g} / \mathrm{mL}$ de NMP. O volume final, para cada orifício, foi de $300 \mu \mathrm{L}$, sendo $230 \mu \mathrm{L}$ padronizados para a adição da cultura bacteriana $10^{5} \mathrm{UFC} \mathrm{mL}^{-1}$, e os $70 \mu \mathrm{L}$ restantes formados por antimicrobiano, cada um dos EPIs e água ultrapura. A redução da MIC, em presença dos EPIs, indica presença de sistemas de efluxo multidrogas. Todos os procedimentos foram realizados em triplicata, juntamente com os controles necessários. E. coli ATCC 25922 foi usada como controle do teste, e E. coli AG100, cepa doada gentilmente pela Dra. Laura McMurry, da Tufts University, EUA, usada como controle positivo dos sistemas de efluxo multidrogas estudados.

\section{RESULTADOS E DISCUSSÃO}

Dos 27 isolados analisados, os isolados de E. coli 13, 21, 24 e $27(14,8 \%)$ apresentaram resistência à ampicilina e ao sulfametoxazol/trimetoprim, simultaneamente, enquanto todos os 27 isolados $(100 \%)$ foram sensíveis ao ceftiofur, à gentamicina, à enrofloxacina e à cefalexina. Embora todos os antimicrobianos testados no trabalho sejam utilizados no tratamento da mastite clínica bovina, o fenótipo de resistência apresentado para ampicilina e sulfametoxazo/trimetoprim somente em quatro isolados indica que os demais antimicrobianos poderiam ser utilizados na terapia da doença nas propriedades onde foram coletadas as amostras. Porém, o uso desses dois antimicrobianos não traduz êxito do tratamento em virtude de inúmeras variáveis existentes in vivo. Além disso, o uso contínuo deles poderá contribuir para a seleção de microrganismos ainda mais resistentes dentro de uma população (Moreira et al., 2008). Rangel e Marin (2009) analisaram a resistência de 231 cepas de E. coli a diferentes antimicrobianos indicados na terapia da mastite bovina, entre esses a ampicilina e o sulfametoxazol/trimetoprim. As cepas apresentaram resistência a diversos antimicrobianos, incluindo ampicilina. Entretanto, os autores observaram sensibilidade ao sulfametoxazol/trimetoprim, o que diverge dos dados obtidos no presente trabalho.

As CIMs foram realizadas para todos os 27 isolados, utilizando-se os dois antimicrobianos, ampicilina e sulfametoxazol/trimetoprim, que compõem o perfil de multirresistência dos quatro isolados de E. coli. Observaram-se valores de CIM para ampicilina variando de $6 \mu \mathrm{g} / \mathrm{mL}$ a 
$250 \mu \mathrm{g} / \mathrm{mL}$ e para sulfametoxazol/trimetoprim de $12 \mu \mathrm{g} / \mathrm{mL}$ a $1500 \mu \mathrm{g} / \mathrm{mL}$. Essas diferenças de CIM para um mesmo antimicrobiano sugerem diferentes perfis fenotípicos de resistência nesses isolados, mesmo sendo obtido de uma mesma fonte.

Houve uma redução das CIMs de ampicilina e sulfametoxazol/trimetoprim tanto nos isolados resistentes (Tab. 1) quanto nos sensíveis (Tab. 2), na presença de PA $\beta \mathrm{N}$ e NMP, o que sugere uma atividade de sistemas de efluxo nesses isolados. Mesmo nos isolados sensíveis, pela metodologia adotada, foi possível detectar sistemas de efluxo, embora a resistência nestes fosse indetectável pelo método do antibiograma. Qualquer alteração nos valores das CIMs (valores abaixo da CIM na ausência de inibidores) dos isolados na presença dos inibidores foi considerada resultado positivo pela metodologia adotada no trabalho.

Tabela 1. Concentração inibitória mínima (CIM) dos antimicrobianos em caldo Muller Hinton, nos isolados de Escherichia coli resistentes, na ausência e presença de PA $\beta$ N e NMP

\begin{tabular}{ccccc}
\multirow{2}{*}{ Isolados } & & \multicolumn{3}{c}{ CIM $\mu \mathrm{g} / \mathrm{mL}$} \\
\cline { 3 - 5 } & Antimicrobianos & Ausência de & \multicolumn{2}{c}{ Presença de inibidores } \\
& & inibidores & PA $\beta \mathrm{N}$ & NMP \\
\hline \multirow{2}{*}{13} & Ampicilina & 250 & 230 & 240 \\
& Sulfametoxazoltrimetoprim & 1500 & 1480 & 1500 \\
21 & Ampicilina & 250 & 220 & 240 \\
& Sulfametoxazoltrimetoprim & 1500 & 1490 & 1500 \\
24 & Ampicilina & 110 & 90 & 110 \\
& Sulfametoxazoltrimetoprim & 1500 & 1490 & 1500 \\
& Ampicilina & 110 & 90 & 110 \\
& Sulfametoxazoltrimetoprim & 1500 & 1480 & 1500 \\
\hline
\end{tabular}

Pelos resultados apresentados nas Tab. 1 e 2 e pelo antibiograma, sugere-se uma atividade de sistemas de efluxo multidrogas, mas devem ser mencionados também outros mecanismos de resistência relacionados à ampicilina e ao sulfametoxazo/trimetoprim que podem estar presentes nesses isolados. Redução da permeabilidade da membrana externa e produção de $\beta$-lactamases AmpC podem atuar em sinergismo com os sistemas de efluxo em E. coli, conferindo resistência a $\beta$-lactâmicos, entre eles a ampicilina (Jacoby, 2009). Alteração da permeabilidade da parede celular, aumento da produção enzimática e da produção de metabólito essencial são alguns mecanismos de resistência bacteriana às sulfas (Acar e Moulin, 2006).

No trabalho realizado por Kern et al. (2006) com E. coli, ao compararem diferentes inibidores, entre eles o NMP e o PA $\beta \mathrm{N}$, os autores observaram que, na presença desses inibidores nas concentrações de $50 \mathrm{mg} / \mathrm{L}$ e $100 \mathrm{mg} / \mathrm{L}$, respectivamente, o $\mathrm{PA} \beta \mathrm{N}$ diminuiu até 16 vezes a CIM de oxacilina, rifampicina, cloranfenicol e claritromicina, naqueles isolados que superexpressaram $a c r A B$ e $a c r E F$, enquanto o NMP diminuiu até quatro vezes. Esses resultados corroboram os do presente estudo devido ao fato de que o inibidor PA $\beta \mathrm{N}$ teve uma melhor atividade que o NMP nos isolados, em relação ao sulfametoxazol/trimetoprim, enquanto para a ampicilina, ambos apresentaram eficiência, embora na presença de $\mathrm{PA} \beta \mathrm{N}$ a diferença dos valores da CIM tenha sido maior (Tab. 2). A diminuição das CIMs para ampicilina em relação ao sulfametoxazol/trimetoprim verificada na presença de $\mathrm{PA} \beta \mathrm{N}$ sugere um sinergismo entre o inibidor e as drogas. Murakami et al. (2006) verificaram que o $\mathrm{PA} \beta \mathrm{N}$ pode competir com alguns antimicrobianos e com outros não, existindo uma relação diferencial entre esse inibidor e o antimicrobiano baseada na natureza do sistema de efluxo e no sítio de ligação do antimicrobiano ao sistema. 
Tabela 2. Concentração inibitória mínima (CIM) dos antimicrobianos em caldo Muller Hinton, de isolados de Escherichia coli sensíveis, na ausência e na presença de PAßN e NMP

\begin{tabular}{|c|c|c|c|c|}
\hline \multirow{3}{*}{ Isolados } & \multirow{3}{*}{ Antimicrobianos } & \multicolumn{3}{|c|}{$\mathrm{CIM} \mu \mathrm{g} / \mathrm{mL}$} \\
\hline & & \multirow[t]{2}{*}{ Ausência de inibidores } & \multicolumn{2}{|c|}{ Presença de inibidores } \\
\hline & & & $\mathrm{PA} \beta \mathrm{N}$ & NMP \\
\hline \multirow[t]{2}{*}{1} & Ampicilina & 6 & 2 & 4 \\
\hline & Sulfametoxazoltrimetoprim & 20 & 14 & 14 \\
\hline \multirow[t]{2}{*}{2} & Ampicilina & 6 & 2 & 4 \\
\hline & Sulfametoxazo/trimetoprim & 22 & 16 & 18 \\
\hline \multirow[t]{2}{*}{3} & Ampicilina & 12 & 4 & 8 \\
\hline & Sulfametoxazoltrimetoprim & 36 & 30 & 30 \\
\hline \multirow[t]{2}{*}{4} & Ampicilina & 10 & 4 & 6 \\
\hline & Sulfametoxazoltrimetoprim & 34 & 28 & 28 \\
\hline \multirow[t]{2}{*}{5} & Ampicilina & 12 & 4 & 6 \\
\hline & Sulfametoxazoltrimetoprim & 36 & 28 & 30 \\
\hline \multirow[t]{2}{*}{6} & Ampicilina & 6 & 2 & 4 \\
\hline & Sulfametoxazoltrimetoprim & 20 & 10 & 12 \\
\hline \multirow[t]{2}{*}{7} & Ampicilina & 6 & 2 & 4 \\
\hline & Sulfametoxazo/trimetoprim & 22 & 8 & 10 \\
\hline \multirow[t]{2}{*}{8} & Ampicilina & 12 & 4 & 8 \\
\hline & Sulfametoxazoltrimetoprim & 26 & 14 & 12 \\
\hline \multirow[t]{2}{*}{9} & Ampicilina & 12 & 2 & 4 \\
\hline & Sulfametoxazo/trimetoprim & 28 & 10 & 14 \\
\hline \multirow[t]{2}{*}{10} & Ampicilina & 6 & 2 & 4 \\
\hline & Sulfametoxazo/trimetoprim & 18 & 10 & 12 \\
\hline \multirow[t]{2}{*}{11} & Ampicilina & 6 & 2 & 2 \\
\hline & Sulfametoxazo/trimetoprim & 12 & 6 & 8 \\
\hline \multirow[t]{2}{*}{12} & Ampicilina & 6 & 2 & 4 \\
\hline & Sulfametoxazo/trimetoprim & 18 & 12 & 12 \\
\hline \multirow[t]{2}{*}{14} & Ampicilina & 8 & 2 & 2 \\
\hline & Sulfametoxazo/trimetoprim & 16 & 10 & 12 \\
\hline \multirow[t]{2}{*}{15} & Ampicilina & 8 & 2 & 4 \\
\hline & Sulfametoxazoltrimetoprim & 28 & 12 & 12 \\
\hline \multirow[t]{2}{*}{16} & Ampicilina & 6 & 2 & 4 \\
\hline & Sulfametoxazo/trimetoprim & 26 & 8 & 10 \\
\hline \multirow[t]{2}{*}{17} & Ampicilina & 12 & 4 & 6 \\
\hline & Sulfametoxazo/trimetoprim & 26 & 10 & 10 \\
\hline \multirow[t]{2}{*}{18} & Ampicilina & 6 & 2 & 4 \\
\hline & Sulfametoxazo/trimetoprim & 28 & 12 & 12 \\
\hline 19 & Ampicilina & 8 & 2 & 2 \\
\hline & Sulfametoxazoltrimetoprim & 30 & 22 & 24 \\
\hline 20 & Ampicilina & 8 & 2 & 4 \\
\hline & Sulfametoxazoltrimetoprim & 32 & 24 & 24 \\
\hline 22 & Ampicilina & 8 & 2 & 2 \\
\hline & Sulfametoxazoltrimetoprim & 30 & 20 & 24 \\
\hline 23 & Ampicilina & 6 & 2 & 4 \\
\hline & Sulfametoxazo/trimetoprim & 28 & 10 & 12 \\
\hline 25 & Ampicilina & 8 & 2 & 6 \\
\hline & Sulfametoxazoltrimetoprim & 32 & 18 & 18 \\
\hline 26 & Ampicilina & 8 & 2 & 4 \\
\hline & Sulfametoxazoltrimetoprim & 30 & 18 & 18 \\
\hline
\end{tabular}




\section{CONCLUSÃO}

Os isolados de E. coli provenientes de leite de bovinos com mastite apresentarem um restrito perfil de resistência a antimicrobianos, contra apenas a ampicilina e o sulfametoxazol/ trimetoprim e, nesses casos, o inibidor PAßN apresentou aumento na atividade desses antimicrobianos sobre os isolados resistentes, revelando-se um promissor candidato a adjuvante no tratamento da mastite.

\section{AGRADECIMENTOS}

À Dra. Laura McMurry, da Tufts University (EUA), pela doação da cepa E. coli AG100; à Fundação de Amparo à Pesquisa em Minas Gerais (Fapemig), ao Conselho Nacional de Desenvolvimento Científico e Tecnológico (CNPq) e à Coordenação de aperfeiçoamento de Pessoal de Nível Superior (Capes), pelo suporte financeiro.

\section{REFERÊNCIAS}

ACAR, J.F.; MOULIN, G. Antimicrobial resistance at farm level. Rev. Sci. Tech., v.25. p.775-792, 2006.

ALTALHI, A.D.; HASSAN, S.A. Bacterial quality of raw milk investigated by Escherichia coli and isolates analysis for specific virulence-gene markers. Food Control, v.20, p.913-917, 2009.

BIGGS, A. Mastitis in cattle. Marlborough: Crowood Press Ltd. Ramsbury, 2009. 192p.

BLUM, B.; HELLER, E.D.; KRIFUCKS, O. et al. Identification of a bovine mastitis Escherichia coli subset. Vet. Microbiol., v.132, p.135-148, 2008.

BRADLEY, A.J.; GREEN, M.J. Factors affecting cure when treating bovine clinical mastitis with cephalosporin-based intramammary preparations. $J$. Dairy Sci., v.92, p.1941-1953, 2009.

CLSI - Clinical and Laboratory Standards Institute. Performance standards for antimicrobial disk and dilution suceptibility tests for bacteria isolated from animals; approved standard. 3. ed. CLSI document M31-A3. Wayne: CLSI press, 2008. 99p.

COSTA, E.O. Binômio: Saúde da glândula mamária e produção leiteira. In: CONGRESSO BRASILEIRO DE BUIATRIA, 8, 2009, Belo Horizonte. Anais... Belo Horizonte: [s.n.] 2009. p.1-20.
FERNANDES, J.B.; ZANARDO, L.G.; GALVAO, N.N. et al. Escherichia coli from clinical mastitis: serotypes and virulence factors. J. Vet. Diagn. Invest., v.23, p.1146-52, 2011.

FONTAINE, M.C.; SMITH, D.G. Microbial biofilms: does breaking the microbes community spirit hold the key to beating persistent mastitis? Vet. J., v.171, p.387-388, 2006.

HUIJPS, K.; HOGEVEEN, H., Preferences of cost factors for mastitis management among Dutch dairy farmers using adaptive conjoint analysis. Prev. Vet. Med., v.92, p.351-359, 2009.

JACOBY, G.A. AmpC beta-lactamases. Clinic. Microbiol. Rev., v.22, p.161-82, 2009.

KERN, W.; STEINKE, P.; SCHUMACHER, A. et al. Effect of 1-(1-naphthylmethyl)-piperazine, a novel putative efflux inhibitor, on antimicrobial drug susceptibility in clinical isolates of Escherichia coli. J. Antim. Chemother., v.57, p.339-343, 2006.

LOMOVSKAYA, O.; BOSTIAN, K.A. Practical applications and feasibility of efflux pump inhibitors in the clinic - A vision for applied use. Biochemical Pharmacol., v.71, p.910-918, 2006.

MATSUMOTO, Y.; HAYAMA, K.; SAKAKIHARA, S. et al. Evaluation of Multidrug Efflux Pump Inhibitors by a New Method Using Microfluidic Channels. PLOS One, v.6, E18547, 2011.

MELCHIOR, M.B.; VAARKAMP, H.; FINKGREMMELS, J. Biofilms: A role in recurrent mastitis infections? Review. Vet. J., v.171, p.398-407, 2006.

MOREIRA, M.A.S.; FERREIRA, A.B.; TRINDADE, T.F.S.L. et al. Resistência a antimicrobianos dependente do sistema de efluxo multidrogas em Escherichia coli isoladas de leite mastítico. Arq. Bras. Med. Vet. Zootec., v.60, p.1307-1314, 2008.

MOREIRA, M.A.S.; OLIVEIRA, J.A.; TEIXEIRA, L.M.; MORAES, C.A. Detection of a chloramphenicol efflux system in Escherichia coli isolated from poultry carcass. Vet. Microbiol., v.109, p.75-81, 2005.

MURAKAMI, S.; NAKASHIMA, R.; YAMASHITA, E. et al. Crystal structures of a multidrug transporter reveal a functionally rotating mechanism. Nature, v.443, p.173-179, 2006.

OLIVER, S.P.; PIGHETTI, G.M.; ALMEIDA, R.A. Mastitis Pathogens. Environmental Pathogens. 2.ed. Knoxville: Academic Press, 2011. 4170p.

PIDDOCK, L.J.V. Clinically relevant chromosomally encoded multidrug resistance efflux pumps in bacteria. Clinical Microbiol. Rev., v.19, p.382-402, 2006 
RANGEL, P.M.; MARIN, J.M. Antimicrobial resistance in Brazilian isolates of shiga toxin-encoding Escherichia coli from cows with mastitis. Ars Vet., v.25, p.18-23, 2009.

RICCI, V.; PIDDOCK, L.J.V. Only for substrate antibiotics is a functional AcrAB-TolC efflux pump and RamA required to select multidrug resistant Salmonella Typhimurium. J. Antimicrob. Chemother., v.64, p.654-657, 2009.

RIBEIRO, M.G.; COSTA, E.O.; LEITE, D.S. et al. Fatores de virulência em linhagens de Escherichia coli isoladas de mastite bovina. Arq. Bras. Med. Vet. Zootec., v.58, p.24-731, 2006.
THORARENSEN, A.; PRESLEY-BODNAR, A.L.; MAROTTI, K.R. et al. 3-Arylpiperidines as potentiators of existing antibacterial agents. Bioorganic Med. Chem. Lett., v.11, p.1903-1906, 2001.

VIVEIROS, M.; MARTINS, M.; COUTO, I. et al. New methods for the identification of efflux mediated MDR bacteria, genetic assessment of regulators and efflux pump constituents, characterization of efflux systems and screening for inhibitors of efflux pumps. Curr. Drug Targets, v.9, p.760-778, 2008.

WEBBER, M.A.; PIDDOCK, L.J.V. The importance of efflux pumps in bacterial antibiotic resistance. $J$. Antimicrob. Chemother., v.51, p.9-11. 2003. 